\title{
Measles-Associated Severe Pneumonia in a Patient with HBeAg-Negative Chronic Hepatitis B: A Case Report
}

\author{
Zhangyan Zhao ${ }^{1}$, Haicheng Tang ${ }^{1, *}$ and Feng $\mathrm{Li}^{1{ }^{1, *}}$
}

\begin{abstract}
Background: Every year, approximately 800,000 people die from liver diseases associated with hepatitis B virus (HBV) infection. Complications outside the liver are common, such as fungal lung infections and viral infections. These complications may be associated with poor immune function, thus making clinical treatment difficult and increasing the risk of death. Therefore, HBVinfection-related liver diseases are worthy of clinical attention and further research.

Case summary: We report a case of $\mathrm{HBeAg}$-negative chronic hepatitis $\mathrm{B}$ in which the patient received entecavir as an anti-HBV treatment after liver dysfunction. During the treatment, the patient was diagnosed with measles and severe viral pneumonia. After comprehensive treatment, including active antiviral medications and mechanical ventilation, the patient recovered and was discharged.
\end{abstract}

Conclusion: HBV infection causes liver damage, affects immune function, and is likely to be associated with viral infections such as measles. Consequently, infections may lead to complications, such as severe viral pneumonia, that endanger patients' lives. To decrease complications and mortality, better understanding of the disease is necessary to enable early diagnosis.

Keywords: CHB, immune function, measles, viral pneumonia, antiviral

\section{Edited by:}

Jian Wu, State Key Laboratory for Diagnosis and Treatment of Infectious

Diseases, National Clinical Research

Center for Infectious Diseases, The First Affiliated Hospital, Zhejiang University

School of Medicine, China

\section{Reviewed by:}

Dawei Cui, The First Affiliated Hospital, Zhejiang University School of Medicine The other reviewer chose to be anonymous

*Corresponding authors: E-mail: thc822@163.com,

Tel: +86-18101607036 (HT);

E-mail: lifeng@shphc.org.cn,

Tel: +86-18121150282 (FL)

${ }^{1}$ Department of Respiratory and Critical Care Medicine, Shanghai Public Health Clinical Center, Fudan University, Shanghai 201508, China

Received: September 032021 Revised: October 022021

Accepted: December 232021

Published Online: January 142022

\section{INTRODUCTION}

Despite advances in the understanding of the immune pathogenesis of chronic hepatitis $\mathrm{B}(\mathrm{CHB})$ in recent years, as well as improvements in diagnosis and treatment methods, $\mathrm{CHB}$ remains associated with substantial morbidity and mortality $[1,2]$. Complications outside of the liver are common, and may include fungal lung infections and viral infections; however, few findings regarding these complications have been reported. Owing to the mutation of the measles virus, the decline in adult measles antibody levels, and the extreme contagiousness of the measles virus, adult measles cases are increasing daily. This article reports the case of a patient with $\mathrm{CHB}$ with measles likely associated with nonstandard treatment, which led to severe viral pneumonia. The case report indicates the need for early introduction of antiHBV therapy to delay or prevent disease progression and complications [3].

\section{CASE PRESENTATION}

The patient, a 39-year-old man, had been vaccinated against measles when he was young, has a history of chronic hepatitis B 
for 20 years. His liver function had been normal in the past, and he was not given antiviral treatment. He was admitted to the hospital on December 17,2016, because his urine had become increasingly yellow over the previous 2 weeks. Tests revealed the following findings: ALT/AST: 1,715/936 U/L, TB/DB: 105/82 $\mu \mathrm{mol} / \mathrm{L}, \mathrm{HBV}-\mathrm{DNA}:>108 \mathrm{IU} / \mathrm{ml}$, and PT: 17 s. The patient had started entecavir and jaundice treatments. Antiviral, liver-protecting, and enzyme-reducing medications had been administered, but his symptoms had not improved, and he was transferred to our hospital for treatment.

\section{PHYSICAL EXAMINATION AT ADMISSION}

The patient was admitted to our hospital on December 30, 2016, he presented with a clear mind, normal calculation ability, altered mental status, and severe yellowing of the skin and sclera; he was negative for liver palm and spider nevus; and his abdomen was soft on palpation, and his liver and spleen were not palpable under the ribs. No edema was observed in either lower limb, and he was negative for flapping tremor. After the patient's admission to our hospital, testing revealed the following:ALT/AST: 238/107 U/L, TB/DB: $440 / 358 \mu \mathrm{mol} / \mathrm{L}$, HBV-DNA: 5.21E+4 IU/ml, PT: 16 s, AFP: >1,210 ng/ml, HBsAg: 6,204 IU/ml, and
HBeAg: negative. After 40 days of hospitalization, retesting indicated the following indices: ALT/AST: 25/58 U/L, TB/DB: 86/75 $\mu \mathrm{mol} / \mathrm{L}, \mathrm{HBV}-\mathrm{DNA}: 951 \mathrm{IU} / \mathrm{ml}$, and AFP: $51.79 \mathrm{ng} / \mathrm{ml}$.

\section{FEVER DURING THE DISEASE COURSE}

The patient began to have a high fever on February 11, 2017. His daily maximum body temperature exceeded $39^{\circ} \mathrm{C}$. After 3 days of fever, a rash appeared, then gradually spread from his face and behind his ears to his chest, back, abdomen, and limbs. The rash was accompanied by a dry cough and chest tightness. However, his liver function, CD4 cells, and lymphocytes were all normal. Chest CT examination revealed diffuse ground-glass density shadows and unclear border patch shadows in both lungs (Fig 1A). Widal's test was negative, anti-rubella virus IgM was negative, and anti-measles virus IgM was positive. He was given ribavirin antiviral treatment. On the sixth day of fever, his chest CT showed a symmetrical map-like distribution of patchy ground-glass shadows in both lungs, with thickening of the interlobular septum; the diffuse lesions in the two lungs had significantly progressed (Fig 1B). The patient was transferred to the respiratory department to continue treatment.
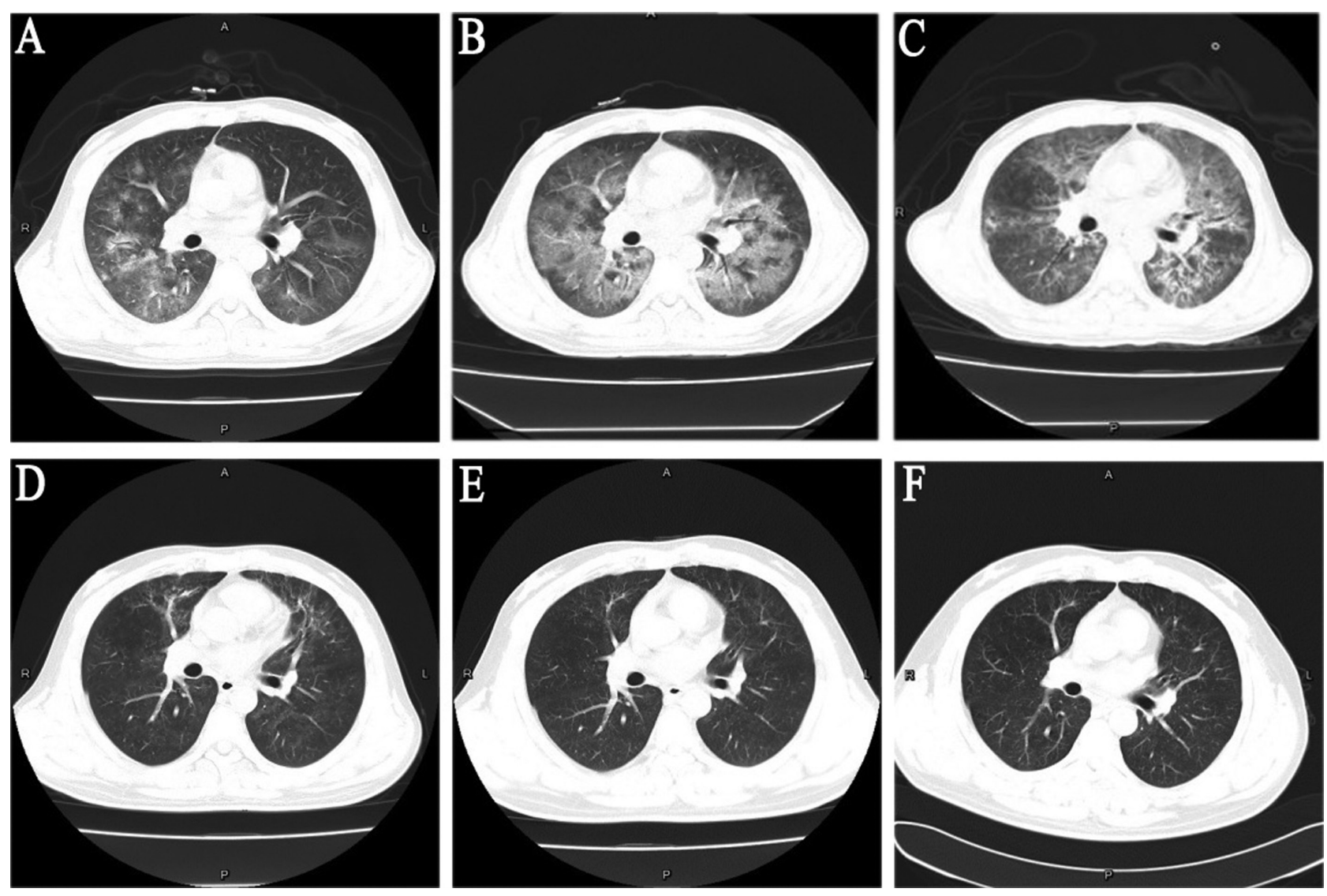

Figure 1 | Chest CT images of the patient. (A) Chest CT findings on the third day after fever. (B) Chest CT findings on the sixth day after fever. (C) Chest CT after removal of tracheal intubation. (D) Chest CT 1 month after discharge. (E) Chest CT three months after discharge. (F) Chest CT 1.5 years after discharge. 


\section{FINAL DIAGNOSIS}

The final diagnosis was viral pneumonia associated with measles, acute respiratory distress syndrome, and $\mathrm{HBeAg-}$ negative $\mathrm{CHB}$.

\section{TREATMENT AND OUTCOME}

At the time of transfer, the patient was short of breath and had a dry cough, fever, and mental weakness. A dark-red scattered rash with lesions of $2-5 \mathrm{~mm}$ had spread over his entire body. On February 21, 2017, his dyspnea was aggravated, his inhaled oxygen concentration was approximately $70 \%$, and his breath rate was $50-55$ breaths per min. The following findings were determined: $\mathrm{pH}: 7.41, \mathrm{PO}_{2}: 8.00$ $\mathrm{kPa}, \mathrm{SaO}_{2}: 91.0 \%$, and oxygenation index $(\mathrm{P} / \mathrm{F}): 86 \mathrm{mmHg}$. His diagnosis had progressed to severe acute respiratory distress syndrome. He was given tracheal intubation, mechanical ventilation, meropenem, ribavirin, and adequate sedation and analgesia. After 3 days, his body temperature returned to normal, and his oxygenation status improved. After 7 days of mechanical ventilation (February 28, 2017), his P/F was $309 \mathrm{mmHg}$, and the ventilator was stopped (chest CT after extubation shown in Fig 1C). At 10 days post-extubation, he was discharged from the hospital with scattered brown pigmented spots over his entire body. Outpatient follow-up chest CT was performed 1 month after discharge (Fig 1D). His HBV-DNA was $<500 \mathrm{IU} / \mathrm{ml} 3$ months after discharge (Fig 1E) and <100 IU/ml 1.5 years after discharge (Fig 1F). His HBV-DNA was followed up until January 5, 2021, and remained below $100 \mathrm{IU} / \mathrm{ml}$ (Fig 2).

\section{DISCUSSION}

Measles is a highly contagious respiratory disease, and almost $100 \%$ of infected people manifest a dominant infection [4]. Epidemiological data have shown that the average annual incidence of measles in China from 2001 to 2016 was 429/100,000 [5-7]. Patients aged 20 to 49 accounted for $67.4 \%$ of the admitted measles patients [8]. In summary, the data suggest that adults over the age of 20 years have low immunity to measles, or the measles antibody level is relatively low in this age group [9]. Our patient had characteristics of measles, such as fever, rash evolution, and measles IgM antibody positivity.

This patient had a history of $\mathrm{CHB}$ and normal liver function at admission to the referring hospital, where antiviral therapy was not initiated. The disease was gradually controlled after entecavir antiviral therapy was initiated. Every year, approximately 800,000 people worldwide die from HBV-infection-associated liver diseases $[1,10]$. In 2018, the

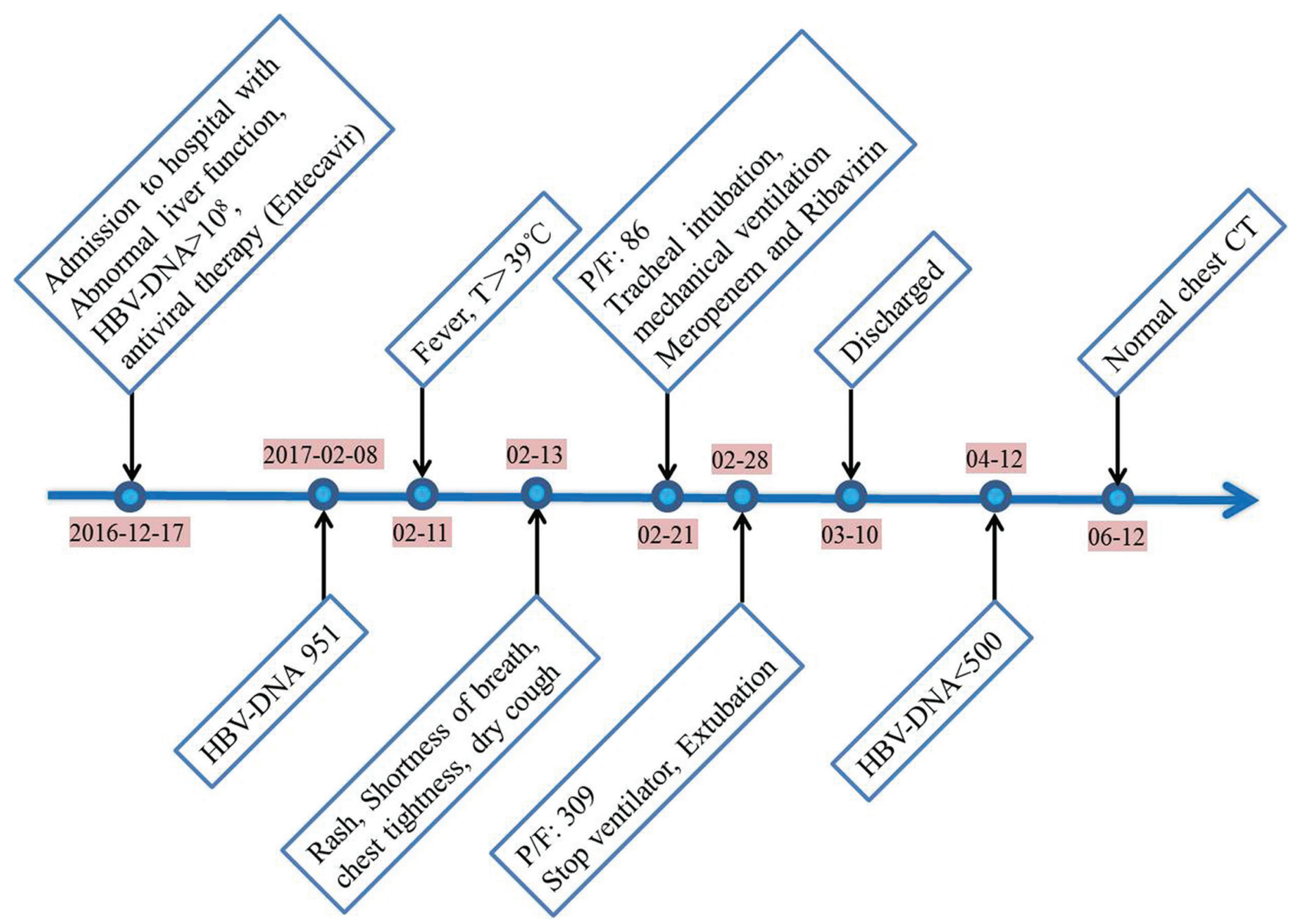

Figure 2 | Disease development and treatment chart. 
American Society of Liver Diseases recommended that adult patients with $\mathrm{CHB}$ and $\mathrm{HBeAg}$-negative immune activity receive unlimited antiviral therapy $[11,12]$. The domestic Guidelines for the Prevention and Treatment of CHB in 2019 [13] recommend that if serology is positive for HBVDNA, the ALT continues to be normal, the patient is older than 30 years of age, and the disease status changes, antiviral therapy should be started.

HBV infection can affect immune function. In patients with $\mathrm{CHB}$, myeloid and plasma-like dendritic cells are diminished, and the maturation of myeloid dendritic cells and the ability of plasma-like dendritic cells to produce interferon- $\alpha$ are impaired. In chronic HBV infection, HBVspecific $\mathrm{T}$ cells become prone to apoptosis, their proliferation ability decreases, and the production of cytokines decreases [13]. Reactivation of HBV infection causes severe liver damage and affects patients' immune function, and consequently may be a risk factor for severe viral pneumonia after measles infection $[14,15]$. In response to the new epidemic trend of adult measles, immunization programs should be strengthened. People who have not received measles vaccination or who have unknown immunization histories should be vaccinated or revaccinated. Patients with suspected measles infections should be isolated and treated in a timely manner.

\section{CONCLUSION}

HBV infection causes liver damage, affects immune function, and is likely to be associated with viral infections such as measles. Understanding of HBV infection should be improved to enable early diagnosis and standardized individualized treatment, to minimize complications and mortality.

\section{ACKNOWLEDGEMENTS}

We thank the frontline medical team of the Shanghai Public Health Clinical Center.

\section{FUNDING}

This work was supported by the Shanghai Municipal Science and Technology Commission (Nos. $20 Z 11901002$ and 21Y11901700 to F-L); Shanghai Municipal Health Commission (No. 202040332 to HC-T); and Shanghai Public Health Clinical Center (No. KY-GW2019-33 to ZY-Z and KY-GW-2021-16 to HC-T).

\section{AVAILABILITY OF DATA AND MATERIALS}

The original source data and materials will be made available upon reasonable request.

\section{ETHICS APPROVAL AND CONSENT TO PARTICIPATE}

This study was approved by the Ethics Committee of the Shanghai Public Health Clinical Center.

\section{CONSENT FOR PUBLICATION}

To publish this case report and all accompanying imaging materials, we obtained written informed consent from close relatives of the patient. A copy of this written informed consent is available for review from the journal's editors. The study was also approved by the Institutional Review Board of the Shanghai Public Health Clinical Center.

\section{COMPETING INTERESTS}

The authors declare that they have no competing interests.

\section{ABBREVIATIONS}

CHB, chronic hepatitis B

DNA, deoxyribonucleic acid

ETV, entecavir

HBeAg, hepatitis B e antigen

HBsAg, hepatitis B surface antigen

$\mathrm{HBV}$, hepatitis B virus

HCC, hepatocellular carcinoma

ALT, alanine aminotransferase

AST, aspartate aminotransferase

AFP, alpha-fetoprotein

$T B$, total bilirubin

$\mathrm{DB}$, direct bilirubin

WHO, World Health Organization

\section{REFERENCES}

1. WHO. Global Hepatitis Report, 2017. https://www.who.int/ publications/i/item/global-hepatitis-report-2017. (accessed 19 April 2017).

2. Dolman GE, Koffas A, Mason WS, Kennedy PT. Why, who and when to start treatment for chronic hepatitis B infection. Curr Opin Virol. 2018;30:39-47.

3. Koffas A, Petersen J, Kennedy PT. Reasons to consider early treatment in chronic hepatitis B patients. Antiviral Res. 2020;177:104783.

4. Wang M, Ma C, Luo H. The protective effect of measles vaccine and its influencing factors. Chinese J Epidemiol. 201435(2):215-217.

5. Shen J, Pan J, Wang Y, Zhao Q, Wang WB. Temporal and spatial distribution characteristics of measles incidence in China from 2001 to 2016. Chin J Epidemiol. 2021;42(4):608-612.

6. Song Q, Su Q, Ma C, Hao LX, Wang HQ. Spatial autocorrelation analysis of measles in China from 2005 to 2014. Chin J Prev Med. 2016;50(7):615-619.

7. Tang X, Yao H, Gao X, Wang J, Tian M, Ding X. Epidemiological characteristics of measles in Jinshan District, Shanghai from 2004 to 2015. Chin J Microbiol Immunol. 2016;36(12):912-917.

8. Zhang M, Yan Y, Tian X, Yang K, Yan Y. Analysis of clinical characteristics of 739 measles patients. Chin J Infect Dis. 2015;(9):555-557.

9. Yao H, Zhao J, Ren J, Tang S, Wang J, Li S, et al. Detection and analysis of measles antibody levels in healthy people in Jinshan District, Shanghai. Chin J Microbiol Immunol. 2017;37(12):910-914.

10. Chinese Medical Association Hepatology Branch, Chinese Medical Association Infectious Diseases Branch. Guidelines for Prevention and Treatment of Chronic Hepatitis B (2015 Update). Chin J Hepatol. 2015;23(12):888-905.

11. Terrault NA, Lok ASF, McMahon BJ, Chang KM, Hwang JP, Jonas $M M$, et al. Update on prevention, diagnosis, and treatment of chronic hepatitis B: AASLD 2018 hepatitis B guidance. Hepatology. 2018;67:1560-1599.

12. Guidelines for the Prevention, Care and Treatment of Persons with Chronic Hepatitis B Infection. Geneva: World Health Organization; 2015.

13. Chinese Medical Association Infectious Diseases Branch, Chinese Medical Association Hepatology Branch. Guidelines for the Prevention and Treatment of Chronic Hepatitis B (2019 Edition). Chin J Hepatol. 2019;27(12):938-961.

14. National Health and Family Planning Commission of the People's Republic of China. Diagnosis of measles. Infectious Disease Information. 2017;30(4):181-189.

15. Jia $C$, Zhao $D$, Zhang $T$, Li X. The imaging manifestations of adult measles virus pneumonia. Chin J Radiol. 2011;45(6):524-526. 